\title{
Prognostic Factors in Breast Cancer: From Staging to the Immu- nohistochemical Profile of Patients with Breast Cancer in a Refer- ence Hospital of Ceara - Brazil
}

\section{Pires Eduardo De Lima Sa ${ }^{1,2 *}$, Morais Livia Mota ${ }^{3}$, Pires Amanda De lima Sa ${ }^{4}$, Matsuda Luis Otavio, Matsuda Josie Budag ${ }^{4}$ and Alves Angelo Roncalli Melo ${ }^{1,2}$}

${ }^{1}$ Department of Medicine, Universidade Estadual do Ceara, Brazil

${ }^{2}$ Department of Medicine, Instituto do Cancer do Ceara, Brazil

${ }^{3}$ Department of Medicine, Universidade Federal do Ceara, Brazil

${ }^{4}$ Department of Medicine, Centro Universitario para o Desenvolvimento do Alto Vale do Itajai, Brazil

*Corresponding author: Pires Eduardo De Lima Sa, Department of Medicine, Instituto do Cancer do Ceara, Papi Júnior street - Rodolfo Teófilo, Fortaleza - CE, 60351-010, Brazil, Tel: 55-(85)-3288-4400

\begin{abstract}
Introduction: Know the tumor stage of breast cancer and identify the immunohistochemical markers are important in the evaluation of predictive and prognostic factors, in the differential diagnosis of breast lesions and in the determination of the possible origin of metastatic neoplasms, besides helping to define the type of treatment.

Methodology: Cross-sectional and quantitative study of patients diagnosed with breast cancer, submitted to treatment at the Ceara Cancer Institute (ICC) mastology service from 2008 to 2012. Describe the main clinical and epidemiological characteristics of breast cancer treated at Haroldo Juacaba Hospital - Ceara Cancer Institute (ICC): Identify types of breast cancer; to evaluate the initial staging, correlating axillary status, local and systemic treatment; to analyze the immunohistochemical profile through receptors standardized by the pathology department of the Hospital, correlating them with the current molecular classification of breast cancer.

Results: A total of 453 medical records were included. $41.5 \%$ of the patients had tumor in the T2 stage, $15.9 \%$ in the T1 stage, $12.6 \%$ in the T4 stage, $11.9 \%$ and in the T3 stage, $11.3 \%$ had tumor in situ. As for lymph nodes, $61.6 \%$ of them were in N0, 24.5\% in N1, $13.0 \%$ in N2 and $0.9 \%$ in N3. $57 \%$ of the patients were sentinel node biopsy, $31 \%$ of the positive frozen. No significant amount of distant metastasis was identified. Regarding tumor type, $89.38 \%$ are ductal, $5.09 \%$ are lobular, $0.88 \%$ are tubular, $0.66 \%$ are mucinous and $7.30 \%$ are other types or mixed. With hormone receptor analysis, we obtained $38.3 \%$ of luminal type A tumors, $32.8 \%$ luminal type B, $21.8 \%$ Triple Negative type and $7.2 \%$ CERB B2 type.
\end{abstract}

Conclusion: Patients were predominantly classified at the IIA clinical stage. The invasive ductal carcinoma subtype is the most prevalent and there is predominance of the luminal molecular pattern, with discrete superiority of luminal pattern A over luminal B.

\section{Keywords}

Breast cancer, Tumor stage, Immunohistochemical markers, Ductal carcinoma, Histopathological classification

\section{Introduction}

It is the most common type of cancer in women worldwide and in Brazil, after non-melanoma skin, accounting for about $28 \%$ of new cases each year. For the biennium 2018-2019, it is estimated that 59,700 new cases of breast cancer have been reported [1]. The prognosis of breast cancer depends on the staging of the disease. When the disease is diagnosed in the beginning, the treatment has greater curative potential [2].

Strategies for the early detection of breast cancer are early diagnosis (approaching people with early signs and/or symptoms of the disease) and screening (applying a test or exam in an apparently healthy asymptomatic population with the goal of identifying lesions suggestive of cancer and, from there, to refer women with altered results for diagnostic investigation and treatment) [3].

Citation: Sa PEDL, Mota ML, Sa PAD, Matsuda OL, Matsuda JB, et al. (2018) Prognostic Factors in Breast Cancer: From Staging to the Immunohistochemical Profile of Patients with Breast Cancer in a Reference Hospital of Ceara - Brazil. Int Arch Med Microbiol 1:005. 
The strategy of early diagnosis contributes to the reduction of the stage of presentation of the cancer, being sometimes known as down-staging. This strategy emphasizes the importance of the education of women and health professionals for the recognition of signs and symptoms of breast cancer, as well as the quick and easy access to health services. It is necessary that the woman be stimulated to seek medical clarification whenever she perceives any suspicious changes in her breasts and to participate in the actions of early detection of breast cancer. The health system needs to be adequate to host, inform and perform the appropriate diagnostic tests in response to this stimulated demand. Priority in the marking of exams should be given to symptomatic women, who already have some suspicious changes in the breast. This strategy proved to be more effective than breast self-examination, that is, most women with breast cancer identified the cancer through occasional palpation compared to self-examination (approximately $65 \%$ of women identify breast cancer casually and $35 \%$ through monthly self-examination). The strategy of early diagnosis is especially important in contexts of advanced presentation of breast cancer [3].

In Brazil, as reviewed in the Guidelines for the Early Detection of Breast Cancer, published in 2015, mammography is the recommended method for tracking the routine of comprehensive health care for women. Mammography is the only examination whose application in screening programs has proven effectiveness in reducing breast cancer mortality and its routine performance is recommended for women aged 50-69 every two years. Mammography in this age group and biennial periodicity are routines adopted in most of the countries that have implemented organized breast cancer screening and are based on the scientific evidence of the benefit of this strategy in reducing mortality in this group and in the favorable balance between risks and benefits. In other age groups and periodicities, the balance between risks and benefits of mammography screening is unfavorable. The success of tracking actions depends on the following pillars: Inform and mobilize the population and organized civil society; Achieve the goal of coverage of the target population; Ensure access to diagnosis and timely treatment; Guarantee the quality of actions; Monitor and continuously manage actions [3].

In this research, however, it was not possible to differentiate the patients diagnosed by screening those diagnosed by disease progression, and it was not possible to obtain the percentage according to the diagnosis form.

It is estimated that in the year 2018 are diagnosed 2200 new cases of breast cancer, an approximate number compared to last year, in which 2160 new cases were diagnosed, most of them in stages III and IV of evolution [4].
In staging, the size of the tumor in the breast (abbreviated as T), the involvement or not of axillary lymph nodes, the lymph node aggression (abbreviated as N) and the presence or absence of evidence of metastases or distant disease (abbreviated as M), known as TNM, as established by the Union for International Cancer Controls (UICC, 2003), a system in use that admittedly offers greater uniformity of criteria adopted by the American Joint Committee on Cancer (AJCC), which considers the clinical and histopathological parameters of the primary tumor and axillary lymph nodes, as well as the presence or absence of metastatic disease [5].

Patient survival and chance of disease recurrence are inversely related to tumor diameter. This, in turn, has a high degree of association with the number of axillary lymph nodes involved. The larger the tumor, the greater the possibility of lymph node metastasis and, in its absence, the tumoral diameter becomes the most important prognostic factor [6].

The number of committed lymph nodes is the most important independent factor of the prognosis, being closely related to the disease free interval and survival. To this end, it must be based on the histopathological study, since in the clinical evaluation $35 \%$ of false results occur; hence the need for axillary evaluation even in early tumors [7].

The advances observed in molecular biology techniques have provided better insight into the mechanisms that regulate cell differentiation and proliferation, which are often recognized and new predictive and prognosis biomarkers are tested in tumor samples by the immunohistochemical method [8]. The expression of hormone receptors [Estrogen Receptors (ERs) and Progesterone Receptors (PR)] and the overexpression or amplification of epidermal human growth factor receptor-2 (HER2) have been identified as important predictive factors among breast cancer patients this research. Currently, these markers are commonly used to define of treatment and establishment of prognosis of the disease, associated with clinical and pathological variables [9].

The objective of this study was to describe the main clinical and epidemiological characteristics of breast cancer patients treated at Haroldo Juacaba Hospital Ceara Cancer Institute (ICC) diagnosed between 2008 and 2012 , to identify the types of breast cancer patients, analyze the immunohistochemical profile of tumors; and to evaluate the initial staging of the patients, correlating to axillary status and to local and systemic treatment.

\section{Methods}

A documentary, cross-sectional and quantitative study was executed of patients with breast cancer diagnosis subjected to treatment at the Haroldo Juacaba Hospital - Ceara Cancer Institute (ICC) in Fortaleza, Ceara. 
Data collection was performed between February and August of 2013, for through the form developed for this study in the patients' medical records, to the rules of the Ceo School of Oncology (ECO), maintained by the ICC.

The data were tabulated with the help of software EPI Info, version 6.04d and analyzed in conjunction with a professional specialized in statistics.

The list of medical records was acquired with the hospital's internal registry service, and the patients subjected to treatment between 2008 and 2012 were selected, with a visit to the mastology service and surgical center.

The records of the registry were requested and those who met the criteria for inclusion were requested: date of diagnosis between 2008 and 2012, SUS patients, diagnosis of breast cancer, and medical records. Data were collected on: The type of tumor and its immunohistochemistry, staging at the time of diagnosis according to the TNM classification and the forms of treatment.

In the data collection form, the following information regarding patients was considered: patients with risk factors for breast cancer, staging, size, types of tumor, anatomopathological aspects, surgical and systemic treatment, metastasis, immunohistochemistry and molecular classification (Appendix A).

The data were tabulated with the aid of the EPI Info software, version $6.04 \mathrm{~d}$ and analyzed in conjunction with a professional specialized in statistics.

This study was approved by the Research Ethics Committee of the Cancer Institute of Ceara - ICC. (Appendix B) under the number of CAAE: 11342512.6.0000.5528.

\section{Results}

Data were collected from 453 medical records of patients with breast cancer between 2008 and 2012. In which the type of tumor and its immunohistochemistry were identified, the staging at the time of diagnosis and the forms of treatment.

On the evaluation of the staging at the moment of the diagnosis it was evidenced:

As to the tumor size at the time of diagnosis, $15.9 \%$ of the patients were in stage $\mathrm{T} 1,41.5 \%$ in stage $\mathrm{T} 2,11.9 \%$ in stage $\mathrm{T} 3,12.6 \%$ in stage $\mathrm{T} 4$, and $11.3 \%$ had tumor in situ.

Regarding the classification of lymph node involvement, $61.6 \%$ were classified as N0, $24.5 \% \mathrm{~N} 1,13.0 \% \mathrm{~N} 2$ and $0.9 \%$ N3.

In addition, according to (Appendix C), 56.95\% of the patients performed sentinel lymph node biopsy (BLNS), $31 \%$ of which were positive congelations.

Regarding tumor type, $89.38 \%$ were ductal; $5.09 \%$, lobular; $0.88 \%$, tubular; $0.66 \%$, mucinous and $7.30 \%$, of other types or mixed.
All the medical records presented the data regarding the study of the immunohistochemistry of the tumors, with the hormonal receptor analysis. Of these, $38.3 \%$ of the tumors were of the Luminal A type, $32.8 \%$ of the Luminal $B$ type, $21.8 \%$ of the triple type Negative and $7.2 \%$ of type B2 CERB.

In the treatment, $99.34 \%$ of the patients underwent a surgical treatment, of which $72.84 \%$ were radical mastectomies. Of the total sample, $75.44 \%$ were subjected to adjuvant radiotherapy (RxT-ADJ), 62.61\% to adjuvant hormone therapy (HxT-ADJ), $53.10 \%$ to adjuvant chemotherapy (QxT-ADJ), $26.55 \%$ to neoadjuvant chemotherapy (QxT-NEO) and $10.40 \%$ to the target therapy.

\section{Discussions}

The precise clinical description and histopathological classification of malignant neoplasms may assist the physician in planning the treatment, facilitating the determination of the prognosis to the patient, assist in the evaluation of treatment results, facilitate the exchange of information between treatment centers, and contribute to the research on human cancer [1].

Internationally, in some developed countries, such as the United States, Canada, the United Kingdom, the Netherlands, Denmark and Norway, there has been an increase in the incidence of breast cancer, accompanied by a reduction in cancer mortality, which is associated with early detection through the introduction of screening mammography and the provision of appropriate treatment [10]. In other countries, as in the case of Brazil, the increase in incidence has been accompanied by an increase in mortality, which can be attributed, mainly, to a delay in diagnosis and the establishment of appropriate therapy.

The survival of women with breast cancer has tripled in the past 10 years, according to a study by the University of Texas MD Anderson Cancer Center, which found that 88 percent of the victims of the disease live at least five years after diagnosis - a decade ago, this percentage was $27 \%$ [11].

This information was passed on in an interview with the agency Efe by Gabriel Hortobagyi (2015), director of the breast cancer research program at the American medical center after a conference on the subject in the Spanish cities of Madrid, Valencia and Barcelona, noting that the control of breast cancer depends on actions of the multiprofessional team of primary health care that aims at the promotion, protection and early diagnosis of the disease.

Tumor size, along with axillary lymph node condition are the two most important prognostic indicators of breast cancer, both being the basis of TNM staging established by the International Union Against Cancer, and are related to the risk of recurrence and the need for radical mastectomy [12]. 
With the advent of mammography and the gradual diffusion of the method, associated to a greater awareness of the population about its importance, it has been observed in some developed countries the significant reduction in the size of tumors at the moment of diagnosis, however, the majority of the patients evaluated in this study presented, at diagnosis, tumors between 2 and $5 \mathrm{~cm}$, stage $T 2$, reflects late diagnosis. In this context, there is a great need to encourage self-examination of the breasts, together with the clinical examination by the gynecologist and the general practitioner in the periodic consultations [13].

The presence of metastases to axillary lymph nodes occurs with great frequency in patients with breast cancer, but most patients did not present palpable lymph nodes at the time of diagnosis, stage NO [13].

The prognosis and relapse worsen as a result of the number of lymph nodes involved, thus, complete axillary emptying was recommended for infiltrating tumors, however, in 1994, the Sentinel Lymph Node technique was developed, which aims to determine the real need for emptying axillary [13].

In this study, $57 \%$ of the patients were performed the Sentinel Lymph node biopsy, with $31 \%$ of the results positive, avoiding unnecessary axillary emptying in 178 patients.

Regarding metastases, in almost all cases, foci were not identified at the distance of the primary tumor.

One of the main prognostic indicators for breast neoplasms is the initial clinical staging. In the present study, there was a predominance of the clinical stage IIA, T2NOMO, at the time of diagnosis, corroborating several studies that indicate the majority of Brazilian patients treated with breast cancer in oncology services present at diagnosis with tumors still bulky.

The study by Thuler and Mendonca [14] analyzed 43,442 cases of breast cancer in the period between 1995 and 2002 and observed that $87.7 \%$ of the women with this diagnosis were found between clinical stages 2 to 4 (stage $2=42.8 \%$, stage $3=32.6 \%$ and stage $4=$ $12.3 \%)$.

In a cross-sectional observational study, Rezende, et al. [13] reported that $51 \%$ of the 104 women studied with suspected or diagnosed breast cancer found between stages 2 and $4(2=28.8 \%, 3=8.7 \%$ and $4=$ $13.5 \%)$.

In countries such as Brazil, where palpable tumors predominate in advanced stages, a reasonable strategy for immediate mortality reduction could be to reduce the time between diagnosis (biopsy) and initiation of treatment (surgical or chemotherapeutic), in order to reduce the number of advanced cases.

Infiltrating ductal carcinoma, in its pure presentation or in combination with other types, is the most common form of breast carcinoma reported in the literature.

In this study, the ductal histological type was found in $89.38 \%$ of the tumors.

In the present study, the analysis of the immunohistochemical profile of the 453 patients allowed the identification and distribution of these profiles in the following percentages: HER overexpressed $7.2 \%$, Luminal A 38.3\%, Luminal B 32.8\%, Basaloid (triple negative) $21.8 \%$, the Claudin-low subtype was not characterized.

The assessment of this profile differs in part from the study of 10,159 women from data from 12 hospital-based cancer registries in several countries (USA, Canada, Europe and Australia), which identified the luminal subtype $A$ as more prevalent followed by the basaloid subtype (negative triple) [15]. This subtype, which in most studies shows a prevalence between 10$20 \%$, in the present study presented with $21.9 \%$.

The treatment is based on the prognostic factors for local and systemic recurrence, which are mainly axillary status, histological type, tumor size, age, degree of histological differentiation, presence of hormonal receptors and the HER-2 [13].

The surgical treatment consists of the approach of the primary tumor and of the axilla, with or without association of the radiotherapy. Indicated for patients without distant metastatic disease [13].

The main factor of the surgical extension corresponds to the tumor volume. The conservative surgeries preserve a good part of the breast, since they are limited to the resection of the area containing the tumor, with a good margin of safety. For this type of surgery, the ideal is that the tumor volume corresponds to a maximum of $20 \%$ of the total breast volume, which is in the early stages and that there is access to complementary radiotherapy, according to the Brazilian Society of Mastology [3].

Radical surgeries are indicated in cases of malignant infiltrating tumors that occupy more than $20 \%$ of the breast volume [3].

The high percentage of radical surgeries performed in this research, $73 \%$, was due to large and locally advanced tumors, being another indication of the late diagnosis of breast cancer.

Radiotherapy has as its main indication the locoregional control of the disease. Its most common use is postoperative (adjuvant radiotherapy), to decrease the chances of relapse, however, in a few cases, it is used in the preoperative (neoadjuvant) to reduce tumor volume. It is also mandatory after conservative surgeries [3].

In the present study, radiotherapy was performed in $75 \%$ of cases, demonstrating the great need of this method. 
Chemotherapy targets local and systemic treatment as well as controlling and treating metastases. It is usually recommended for patients with infiltrating tumors, positive lymph nodes or when the tumor is large, reducing it to allow conservative surgeries [13]. Like radiotherapy, it may be adjuvant or neoadjuvant. It was performed, respectively, in about $53 \%$ of patients adjuvant and $26 \%$ as neoadjuvant.

Hormone receptors, when present in at least $10 \%$ of the cells, are important predictive factors in the prognosis of women with breast cancer. Its presence correlates with the benefit of hormone therapy, which uses substances that inhibit the activity of endogenous hormones (estrogen and progesterone) on the breast [13].

The hormone therapy was performed in approximately $63 \%$ of patients in this study.

Considering the current implications in the therapeutic approach to breast cancer, studies should be encouraged to provide a better understanding of the selection of the most appropriate markers to be used in clinical practice in our country, as well as a better understanding of the distribution of breast cancer disease in Brazilian women.

It is very important to detect breast cancer early because of its lethality, as well as to the physical and emotional sequelae for the woman when receiving this diagnosis, allowing greater survival and better quality of life for women.

It is hoped that this research may contribute to the knowledge of professionals and health managers regarding the epidemiological, clinical and pathological aspects of breast neoplasm in the State of Ceara, comparing them with national and international data, favoring a better elaboration and application of measures early detection, diagnostic and therapeutic methods.

\section{Acknowledgments}

We appreciate the collaboration of Haroldo Juacaba Hospital and its employees for the availability and cordiality in facilitating this research, but we are especially grateful to the patients for having allowed us to enter into the intimacy of their health states so that the study could be carried out.

\section{Conflicts of Interest}

The authors declare that there are no conflicts of interest.

\section{References}

1. INCA (2018) Cancer statistics. National Cancer Institute, Rio de Janeiro, Brazil.

2. INCA (2013) Cancer statistics. National Cancer Institute, Rio de Janeiro, Brazil.

3. Migowski A, Stein AT, Ferreira CBT, Ferreira DMTP, Nadanovsky $P$ (2018) Diretrizes para deteccao precoce do cancer de mama no Brasil. I - Metodos de elaboracao. Cadernos de Saude Publica 34: e00116317.

4. Instituto Nacional de Cancer Jose Alencar Gomes da Silva (2017) Estimativa 2018: Incidencia de cancer no Brasil Coordenacao de Prevencao e Vigilancia - Rio de Janeiro: INCA.

5. Barros ACSD, Buzaid AC (2007) Breast Cancer. Cancer de mama: Tratamento multidisciplinary. ( $1^{\text {st }}$ edn $)$, Dentrix Edicao e Desing, Sao Paulo.

6. Boff RA, Wisintainer F (2006) Modern mastology: A multidisciplinary approach. ( $1^{\text {st }}$ edn $)$, Caxias do Sul, Brazil.

7. Harris JR, Lippman ME, Morrow M, Osborne CK (2004) Diseases of the breast. Philadelphia, New York.

8. Jemal A, Bray F, Center MM, Ferlay J, Ward E, et al. (2011) Global cancer statistics. CA Cancer J Clin 61: 69-90.

9. Guerra MR, Gallo CDM, Mendonca GA, Silva GA (2005) Risk of cancer in Brazil: Trends and more recent epidemiological studies. Rev Bras Cancerol 51: 227-234.

10. INCA (2004) Controlof breast cancer, Consensus document. National Cancer Institute, Rio de Janeiro, Brazil.

11. Almudena D (2013) Survival of women with breast cancer triples in ten years. Madrid.

12. Abreu A, Koifman S (2002) Fatores prognosticos no cancer da mama feminina. Revista Brasileira de Cancerologia 48: 113-131.

13. Rezende MCR, Koch HA, Figueiredo JA, Thuler (2009) LCS Causes of delay in the diagnostic confirmation of breast lesions in women attended at a reference center of the Unified Health System in Rio de Janeiro. Rev Bras Ginecol Obstet 31: 75-81.

14. Thuler LC, Mendonca GA (2005) Initial staging of breast and cervical cancer cases in Brazilian women. Rev Bras Ginecol Obstet 27: 656-660.

15. Blows FM, Driver KE, Schmidt MK, Broeks A, Van Leeuwen FE, et al. (2010) Subtyping of breast cancer by immunohistochemestry to investigate the relationship between subtype and short and long term survival: A collaborative analysis of data for 10,159 cases fron 12 studies. PLoS Med 7: e1000279. 